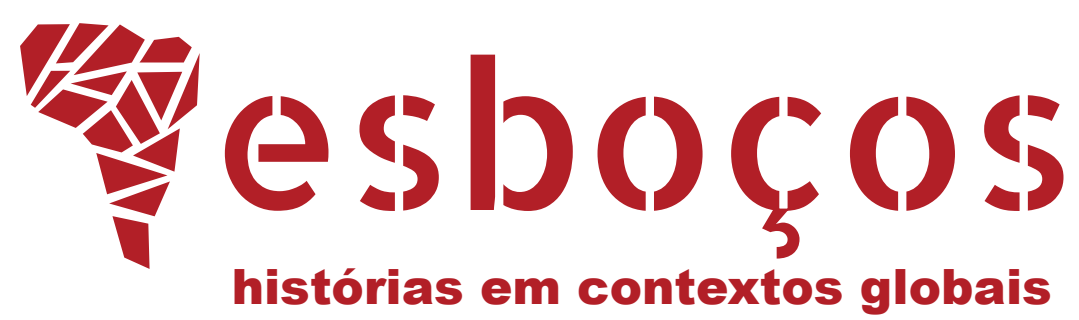

\title{
LISTA ALFABÉtICA DOS \\ PARECERISTAS QUE COLABORARAM \\ COM A ESBOCGOS: HISTÓRIAS EM \\ CONTEXTOS GLOBAIS EM 2021: \\ VOLUME 28, NÚMEROS 47, 48 E 49
}

Alphabetical list of reviewers who contributed to Esboços: histories in

global contexts in 2021: Volume 28, Numbers 47, 48, and 49

Flávia Florentino Varella a,b

(1) https://orcid.org/0000-0001-7123-8807

E-mail: flavia_varella@hotmail.com

Victor Leão Wobeto ${ }^{\mathrm{a}, \mathrm{d}}$

(D) https://orcid.org/0000-0003-1443-0338

E-mail: victorlwobeto@gmail.com

a Universidade Federal de Santa Catarina, Centro de Filosofia e Ciências Humanas,

Departamento de História, Florianópolis, SC, Brasil

${ }^{\mathrm{b}}$ Editora-chefe de Esboços: histórias em contextos globais

d Estagiário de Esboços: histórias em contextos globais 
Abbas Jong, Humboldt-Universität zu Berlin, Berlim, Alemanha

Adelaide Maria Muralha Vieira Machado, Universidade de São Paulo, São Paulo, SP, Brasil

Agnès Pellerin, Université Paris 8 Vincennes-St Denis, Saint-Denis, França

Alain El Youssef, Universidade de São Paulo, São Paulo, SP, Brasil

Alessandro Batistella, Universidade de Passo Fundo, Passo Fundo, RS, Brasil

Alexandre Andrade da Costa, Fundação Educacional de Fernandópolis,

Fernandópolis, SP, Brasil

Alexandre Maccari Ferreira, Universidade Federal de Santa Maria, Santa Maria, RS, Brasil

Aline dos Santos Lima, Instituto Federal Baiano, Santa Inês, BA, Brasil

Aline Duarte da Graça Rizzo, Instituto de Pesquisa Econômica Aplicada, Brasília, DF, Brasil

Amilcar Torrão Filho, Pontifícia Universidade Católica de São Paulo, São Paulo, SP, Brasil

Ana Maria Alves, Instituto Politécnico de Bragança, Bragança, Portugal

Andra Brosy Chastain, Washington State University, Pullman, Estados Unidos

André Fabiano Voigt, Universidade Federal de Uberlândia, Uberlândia, MG, Brasil

André Luiz Morais Zuzarte Bravo, Fundação Casa de Rui Barbosa, Rio de Janeiro, RJ, Brasil

Andrea Mariuzzo, Scuola Normale Superiore, Pisa, Itália

Anne Garland Mahler, University of Virginia, Charlottesville, Estados Unidos

Anne Rush, University of Maryland, College Park, Estados Unidos

Antonio Gámiz Gordo, Universidad de Sevilla, Sevilha, Espanha

Aristeu Elisandro Machado Lopes, Universidade Federal de Pelotas, Pelotas, RS, Brasil

Arno Sonderegger, Universität Wien, Viena, Áustria 
Beatriz Juana Bissio Staricco Neiva Moreira, Universidade Federal do Rio de Janeiro, Rio de Janeiro, RJ, Brasil

Carlile Lanzieri Júnior, Universidade Federal de Mato Grosso, Cuiabá, MS, Brasil

Carolina Marotta Capanema, Universidade Federal de Viçosa, Viçosa, MG, Brasil

Carolina Junqueira, Universidade de São Paulo, São Paulo, SP, Brasil

Case Watkins, James Madison University, Harrisonburg, VA, Estados Unidos

Celine Ugolini, University of New Orleans, Nova Orleans, LA, Estados Unidos

Charles Monteiro, Pontifícia Universidade Católica do Rio Grande do Sul, Porto Alegre, RS, Brasil

Clarissa Franzoi Dri, Universiade Federal de Santa Catarina, Florianópolis, SC, Brasil

Daniel Pinha Silva, Universidade do Estado do Rio de Janeiro, Rio de Janeiro, RJ, Brasil

Diego Galeano, Pontifícia Universidade Católica do Rio de Janeiro, Rio de Janeiro, RJ, Brasil

Êça Pereira da Silva, Universidade Federal do Tocantins, Palmas, TO, Brasil

Eliane Cristina Deckmann Fleck, Universidad de Buenos Aires, Buenos Aires, Argentina

Emilio de Antuñano, Trinity University, San Antonio, Estados Unidos

Fábio da Silva Sousa, Universidade Federal de Mato Grosso do Sul, Campo Grande, MS, Brasil

Fabrício Antônio Antunes Soares, Universidade Estadual de Londrina, Londrina, PR, Brasil

Felipe Paiva Soares, Universidade Federal Fluminense, Niterói, RJ, Brasil

Filipe Queiroz de Campos, Universidade Federal de Juiz de Fora, Juiz de Fora, MG, Brasil

Flávia Cesarino Costa, Universidade Federal de São Carlos, São Carlos, SP, Brasil

Flavia Fernandes de Souza, Universidade Federal Fluminense, Niterói, RJ, Brasil 
Francisco Carlos Jacinto Barbosa, Universidade Estadual do Ceará, Fortaleza, CE, Brasil

Francisco Carlos Palomanes Martinho, Universidade de São Paulo, São Paulo, SP, Brasil

Frederico Ágoas, Universidade Nova de Lisboa, Lisboa, Portugal

Gilles Paché, Aix-Marseille Université, Aix-en-Provence, França

Glaucia Cristina Candian Fraccaro, Universidade Federal de Santa Catarina, Florianópolis, SC, Brasil

Gustavo Soranz Gonçalves, Centro Universitário Fametro, Manaus, AM, Brasil

Haila Manteghi Amin, Westfälische Wilhelms-Universität Münster, Münster, Alemanha

Helen Scorsatto Ortiz, Instituto Federal de Educação, Ciência e Tecnologia do Rio Grande do Sul, Porto Alegre, RS, Brasil

Hugo Antonio Fazio Vengoa, Universidad de los Andes, Bogotá, Colômbia

Jacqueline Ly, Yale University, New Haven, Estados Unidos

Jan Hansen, Humboldt Universität zu Berlin, Berlim, Alemanha

Jayme Lúcio Fernandes Ribeiro, Instituto Federal de Educação, Ciência e Tecnologia do Rio de Janeiro, Duque de Caxias, RJ, Brasil

Joana de Moares Monteleone, Universidade de São Paulo, São Paulo, SP, Brasil João Klug, Universidade Federal de Santa Catarina, Florianópolis, SC, Brasil Johnni Langer, Universidade Federal da Paraíba, João Pessoa, PB, Brasil José Augusto Miranda, Instituto Brasileiro de Mercado de Capitais, Rio de Janeiro, RJ, Brasil

José Carlos Vilardaga, Universidade Federal de São Paulo, Guarulhos, SP, Brasil José Lins Duarte, Universidade Federal do Piauí, Teresina, PI, Brasil Juliana Manzoni Cavalcanti, Universidade de São Paulo, São Paulo, SP, Brasil Júlio Barnez Pignata Cattai, Instituto de Educação e Ensino Superior de Campinas, Campinas, SP, Brasil

Karina Kriegesmann, Freie Universität Berlin, Berlim, Alemanha 
Karine Varley, University of Strathclyde, Glasgow, Reino Unido

Karl Schurster Veríssimo de Sousa Leão, Universidade de Pernambuco, Recife, PE, Brasil

Kasper Braskén, Åbo Akademi University, Turku, Finlândia

Leandro Calbente Câmara, Pesquisador autônomo, Brasil

Leandro Duarte Rust, Universidade de Brasília, Brasíla, DF, Brasil

Licínio Miranda, University of Florida, University of Florida, Gainesville, Estados Unidos

Lindener Pareto Júnior, Pontifícia Universidade Católica de Campinas, Campinas, SP, Brasil

Lise Fernanda Sedrez, Universidade Federal do Rio de Janeiro, Rio de Janeiro, RJ, Brasil

Luciana de Oliveira Dias, Universidade Federal de Goiás, Goiânia, GO, Brasil

Luis Guilherme Assis Kalil, Universidade Federal Rural do Rio de Janeiro, Nova Iguaçu, RJ, Brasil

Luis Ruiz Padrón, Universidad de Málaga, Málaga, Espanha

Luíza Beatriz Amorim Melo Alvim, Universidade Federal do Rio de Janeiro, Rio de Janeiro, RJ, Brasil

Marcelo de Sousa Neto, Universidade Estadual do Piauí, Teresina, PI, Brasil

Marcelo Rosanova Ferraro, Brown University, Providence, Estados Unidos

Márcia Regina Romeiro Chuva, Universidade Federal do Estado do Rio de Janeiro, Rio de Janeiro, RJ, Brasil

Marcos Gerhardt, Universidade de Passo Fundo, Passo Fundo, RS, Brasil

Maria do Rosário Rolfsen Salles, Universidade Anhembi Morumbi, São Paulo, SP, Brasil

Mariana Rangel Joffily, Universidade do Estado de Santa Catarina, Florianópolis, SC, Brasil

Mariana Libânio de Rezende Dantas, Johns Hopkins University, Baltimore, Estados Unidos 
Martha Campos Abreu, Universidade Federal Fluminense, Niterói, RJ, Brasil

Mateus Henrique de Faria Pereira, Universidade Federal de Ouro Preto, Mariana, MG, Brasil

Mercedes García Ferrari, Universidad Nacional de General Sarmiento, Malvinas Argentinas, Argentina

Michel Gherman, Universidade Federal de Santa Catarina, Florianópolis, SC, Brasil

Monique Sochaczewski Goldfeld, Instituto Brasileiro de Ensino, Desenvolvimento e Pesquisa, São Paulo, SP, Brasil

Murillo Dias Winter, Universidade de São Paulo, São Paulo, SP, Brasil

Myrian Sepúlveda dos Santos, Universidade do Estado do Rio de Janeiro, Rio de Janeiro, RJ, Brasil

Nikelen Acosta Witter, Universidade Federal de Santa Maria, Santa Maria, RS, Brasil

Nino Vallen, Freie Universität Berlin, Berlim, Alemanha

Odair da Cruz Paiva, Universidade Federal de São Paulo, Guarulhos, SP, Brasil

Odilon Caldeira Neto, Universidade Federal de Juiz de Fora, Juiz de Fora, MG, Brasil

Paula Chamy Pereira da Costa, Universidade Estadual de Campinas, Campinas, SP, Brasil

Paula Rafaela da Silva, Pontifícia Universidade Católica do Rio Grande do Sul, Porto Alegre, RS, Brasil

Pedro Afonso Cristovão dos Santos, Universidade Federal da Integração LatinoAmericana, Foz do Iguaçu, PR, Brasil

Pedro Jimenez Cantisano, University of Nebraska, Omaha, Estados Unidos

Pedro Henrique Pedreira Campos, Universidade Federal Rural do Rio de Janeiro, Seropédica, RJ, Brasil

Rafael Simone Nharreluga, Universidade Eduardo Mondlane, Maputo, Moçambique

Raissa Brescia dos Reis, Universidade Federal do Rio de Janeiro, Rio de Janeiro, RJ, Brasil

Raquel Schefer, Université de Paris III Sorbonne-Nouvelle, Paris, França

Raul da Silva Ventura Neto, Universidade Federal do Pará, Belém, PA, Brasil, 
Renato Júnio Franco, Universidade Federal Fluminense, Niterói, RJ, Brasil

Ricardo Sequeiros Coelho, Universidade do Porto, Porto, Portugal

Robert Kramm, Ludwig-Maximilians-Universität München, Munique, Alemanha

Rodrigo Octavio D’Azevedo Carreiro, Universidade Federal de Pernambuco, Recife, PE, Brasil

Rodrigo Perla Martins, Universidade FEEVALE, Novo Hamburgo, RS, Brasil

Rodrigo Turin, Universidade Federal do Estado do Rio de Janeiro, Rio de Janeiro, RJ, Brasil

Ryan Thomas Devlin, Columbia University, Nova Iorque, Estados Unidos

Sabrina Steinke, Universidade Federal do Piauí, Teresina, PI, Brasil

Samira Peruchi Moretto, Universidade Federal da Fronteira Sul, Chapecó, SC, Brasil

Stella Paresa Krepp, Universität Bern, Berna, Suiça

Steven Eric Byrd, University of New England, Biddeford, Estados Unidos

Telma Maria Gonçalves Menicucci, Universidade Federal de Minas Gerais, Belo Horizonte, MG, Brasil

Theo Williams, Durham University, Durham, Reino Unido

Thiago Juarez Ribeiro da Silva, Universidade de São Paulo, São Paulo, SP, Brasil

Thiago Nascimento Krause, Universidade Federal do Estado do Rio de Janeiro, Rio de Janeiro, RJ, Brasil

Valéria Aparecida Alves, Universidade Estadual do Ceará, Fortaleza, CE, Brasil

Vitor Eduardo Schincariol, Fundação Universidade Federal do ABC, Santo André, SP, Brasil

Vitor Izecksohn, Universidade Federal do Rio de Janeiro, Rio de Janeiro, RJ, Brasil

Wagner Pinheiro Pereira, Universidade Federal do Rio de Janeiro, Rio de Janeiro, RJ, Brasil

Washington Santos Nascimento, Universidade do Estado do Rio de Janeiro, Rio de Janeiro, RJ, Brasil 


\section{NOTAS DE AUTOR}

\section{AUTORIA}

Flávia Florentino Varella: Doutora. Professora adjunta, Universidade Federal de Santa Catarina, Centro de Filosofia e Ciências Humanas, Departamento de História, Florianópolis, SC, Brasil.

Victor Leão Wobeto: Graduando, Universidade Federal de Santa Catarina, Centro de Filosofia e Ciências Humanas, Departamento de História, Florianópolis, SC, Brasil.

\section{ENDEREÇO PARA CORRESPONDÊNCIA}

Flávia Florentino Varella. Departamento de História, CFH, Bloco E, $6^{\circ}$ andar, Campus Universitário da UFSC, Trindade, 88040-970, Florianópolis, SC, Brasil.

\section{LICENÇA DE USO}

(c) Flávia Florentino Varella e Victor Leão Wobeto. Esta lista de pareceristas está licenciada sob a Licença Creative Commons CC-BY. Com essa licença você pode compartilhar, adaptar, criar para qualquer fim, desde que atribua a autoria da obra.

\section{PUBLISHER}

Universidade Federal de Santa Catarina. Programa de Pós-Graduação em História. Portal de Periódicos UFSC. As ideias expressadas neste artigo são de responsabilidade de seus autores, não representando, necessariamente, a opinião dos editores ou da universidade.

\section{EDITORES}

Flávia Florentino Varella (Editora-chefe) 